\title{
Skipping orbits and enhanced resistivity in large-diameter InAs/GaSb antidot lattices
}

\author{
J. Eroms, ${ }^{*}$ M. Zitzlsperger, and D. Weiss \\ Universität Regensburg, D-93040 Regensburg, Germany \\ J. H. Smet and C. Albrecht \\ Max-Planck-Institut für Festkörperforschung, Heisenbergstraße 1, D-70569 Stuttgart, Germany \\ R. Fleischmann \\ Max-Planck-Institut für Strömungsforschung, Bunsenstraße 10, D-37073 Göttingen, Germany
}

M. Behet, J. De Boeck, and G. Borghs

IMEC, Kapeldreef 75, B-3001 Leuven, Belgium

(Received 27 July 1998)

\begin{abstract}
We investigated the magnetotransport properties of high-mobility InAs/GaSb antidot lattices. For samples with large antidot diameter, we found a broad maximum of classical origin around $2.5 \mathrm{~T}$ in addition to the usual commensurability features at low magnetic fields. The broad maximum can be ascribed to a class of orbits involving multiple reflections on a single antidot. This is shown by both a simple transport calculation based on a classical Kubo formula and an analysis of the Poincare sections at different magnetic-field values. At low temperatures we observe weak $1 / B$-periodic oscillations superimposed on the classical maximum. [S0163-1829(99)50512-2]
\end{abstract}

Periodically modulated two-dimensional electron gases (2DEG's) offer the possibility to study electron motion in artificially tailored periodic potentials. ${ }^{1}$ If the modulation potential is strong enough to deplete parts of the 2DEG around the potential maxima the system is called an antidot (AD) array. Antidot lattices are model systems to study both the classical chaotic motion of electrons in the potential landscape as well as resulting band-structure effects. ${ }^{1}$ Investigations so far have essentially focused on the low field regime where the low-temperature magnetoresistance reflects peaks whenever the classical cyclotron diameter $2 R_{c}$ fits around a (geometry-dependent) specific number of antidots. ${ }^{2}$ The last maximum appears in the resistivity when $2 R_{c}$ is equal to the period $a$ of the array. Here we focus on a structure appearing at slightly higher fields corresponding to $B$ values where $2 R_{c}<a$ holds. While antidot lattices are usually based on $\mathrm{GaAs} / \mathrm{Al}_{x} \mathrm{Ga}_{1-x} \mathrm{As}$ heterojunctions, we used here the material system InAs/GaSb to fabricate antidot arrays. Due to the pinning of the Fermi energy within the conduction band at open surfaces of InAs, ${ }^{3}$ depletion regions around the antidots should be significantly smaller than in GaAs based systems. This should allow, due to the resulting steep potential posts, to fabricate very short period antidot arrays or, as is the case here, the fabrication of large antidots with small constrictions between neighboring antidots.

Our samples are fabricated from InAs/GaSb heterostructures grown on undoped GaAs substrates by molecular beam epitaxy. The epitaxial layers on the substrate consist of a 1.1 $\mu \mathrm{m}$ AlGaSb layer, a $0.5 \mu \mathrm{m}$ thick GaSb buffer followed by a ten-period AlSb/GaSb superlattice, a GaSb barrier of 50 $\mathrm{nm}$ thickness, a $15 \mathrm{~nm}$ InAs quantum well, and a $5 \mathrm{~nm} \mathrm{GaSb}$ top layer (see the upper inset in Fig. 1). While the heterostructure is nominally undoped, the carrier concentration $n_{s}$ can be adjusted by the top layer thickness. ${ }^{4-6}$ After pattern- ing of Hall bars with photolithography and wet chemical etching $^{7}$ and the preparation of evaporated indium contacts, the samples show a carrier density of about $n_{s}=1.5 \times 10^{12}$ $\mathrm{cm}^{-2}$ and a mobility of about $\mu=300000 \mathrm{~cm}^{2} / \mathrm{V}$ s. This corresponds to a mean free path of $6 \mu \mathrm{m}$, which is much larger than the lattice period $a=380 \mathrm{~nm}$, thus making commensurability features observable. The antidots were defined with electron beam lithography and etched $35 \mathrm{~nm}$ deep with the solution described above. A scanning electron micrograph of the samples shows the antidots to be uniform in size and

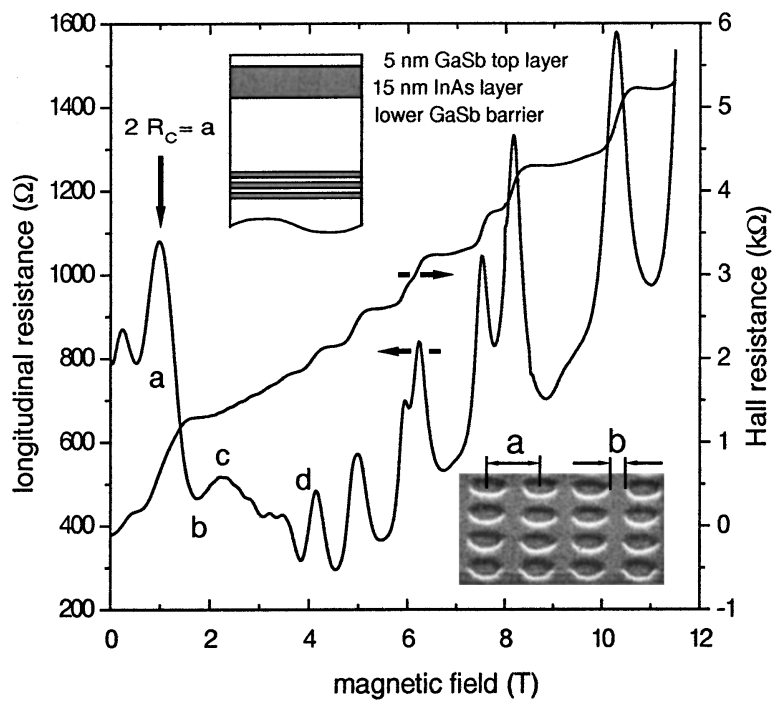

FIG. 1. Magnetoresistance and Hall resistance of an InAs/GaSb antidot lattice. Lower inset: A scanning electron microscopy picture of the sample. Upper inset: Layer sequence of the heterostructure as described in the text. Letters a to d denote $B$-field positions of interest, which are analyzed numerically in Fig. 4. 
shape with an AD diameter of $250 \mathrm{~nm}$. In order to determine which features are caused by the large AD diameter, we also fabricated a number of samples with periods from $a=300$ $\mathrm{nm}$ to $a=1000 \mathrm{~nm}$, but with small AD diameters of about $100 \mathrm{~nm}$ to $150 \mathrm{~nm}$.

The four-point magnetotransport measurements were carried out at temperatures ranging from $1.5 \mathrm{~K}$ to $40 \mathrm{~K}$ using standard lock-in techniques. Utilizing the negative persistent photoconductivity of InAs quantum wells, ${ }^{8}$ the carrier density could be varied by illuminating the sample with a red light-emitting diode (LED). The magnetoresistance and Hall resistance traces at a carrier density of $1.33 \times 10^{12} \mathrm{~cm}^{-2}$ and a temperature of $1.5 \mathrm{~K}$ are shown in Fig. 1. At low fields two commensurability peaks in the magnetoresistance curve can be observed. These well-known maxima were also found in the control samples with small AD diameter (not shown here). The peak at about $1 \mathrm{~T}$ belongs to trajectories going around one antidot, the other one at $0.25 \mathrm{~T}$ corresponds to a deformed cyclotron orbit around four antidots. In the simplest model, the enhanced resistivity at these field values can be ascribed to electrons pinned on commensurate cyclotron orbits, which cannot carry current. ${ }^{2,9}$ At fields above $3.5 \mathrm{~T}$, strong Shubnikov-de Haas $(\mathrm{SdH})$ oscillations appear and from $6 \mathrm{~T}$, spin splitting of the Landau levels is resolved. By comparison to a Hall-bar sample without AD's, we can conclude that the quality of the 2DEG was not seriously affected by the AD patterning process. For large diameter AD's, we find an unusual broad peak structure in the region between 1.5 $\mathrm{T}$ and $3.5 \mathrm{~T}$ whose origin will be discussed below. In earlier work, a corresponding shoulder was ascribed to etchinduced defects. ${ }^{10}$ For a sample with triangular AD's, a highfield shoulder at $2 R_{c}=a / 2$ was explained by electrons being reflected on the straight $\mathrm{AD}$ edges. ${ }^{11} \mathrm{We}$ will show below that the extra structure can be ascribed to electron trajectories skipping around one antidot. This type of orbit also proved to be relevant in a recent focusing type experiment. ${ }^{12}$

Figure 2 shows the dependence of the magnetoresistance traces on the carrier density (changed by illumination) and temperature (inset). The unusual peak structure shifts similar to the main commensurability maximum at $2 R_{c}=a$. This suggests, together with the weak temperature dependence of the peaks (see lower inset of Fig. 2), a classical origin of the phenomenon. Associating the magnetic-field value at which the broad maxima start to appear (marked by arrows) with the matching condition $R_{c}=b$ we find a value of $b=110 \mathrm{~nm}$. This value $b$ corresponds to the distance between neighboring antidots in our device. This finding is consistent with the picture that the broad maximum is associated with rosetteshaped orbits skipping around one antidot. These trajectories become important for $R_{c} \sim b$.

In order to clarify the origin of the classical maximum between $1.5 \mathrm{~T}$ and $3.5 \mathrm{~T}$ we carried out numerical calculations following recent work. ${ }^{9}$ We evaluated the Kubo formula ${ }^{13}$

$$
\sigma_{i j}=\frac{e^{2} m^{*}}{\pi \hbar^{2}} \int_{0}^{\infty} d t e^{-t / \tau}\left\langle v_{i}(t) v_{j}(0)\right\rangle
$$

to calculate the conductivity tensor $\sigma_{i j}$ where the indices $i$ and $j$ stand for the $x$ direction and $y$ direction, respectively. The trajectories and the resulting velocities $v_{i}$ and $v_{j}$ were

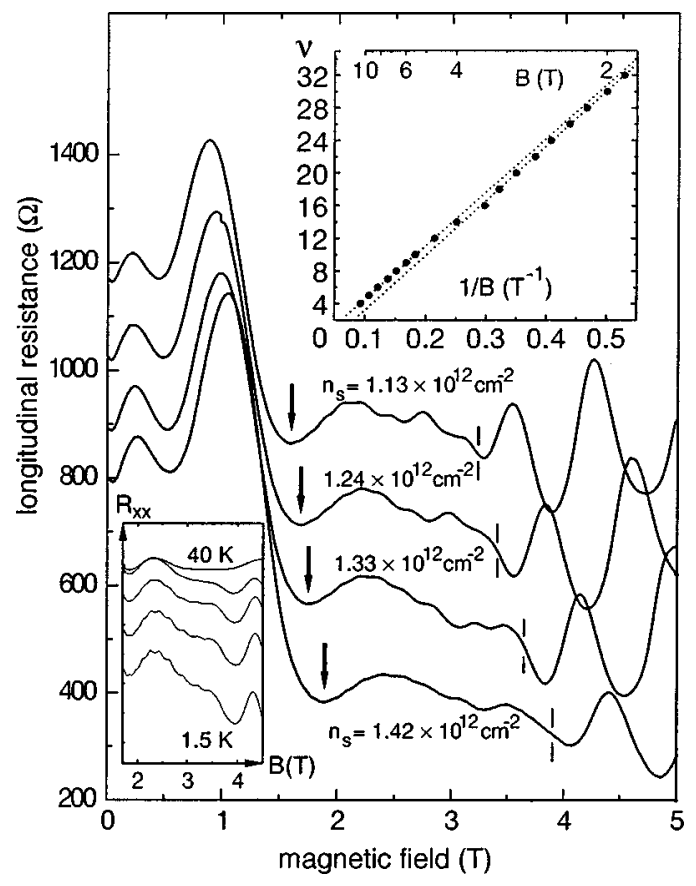

FIG. 2. Density dependence of the magnetoresistance. Arrows indicate the beginning of the new structure (at $R_{c} \sim b$ ), vertical lines mark the onset of strong SdH oscillations (at $2 R_{c} \sim b$ ). Lower inset: Temperature dependence of the features at $n_{s}=1.42 \times 10^{12} \mathrm{~cm}^{-2}$. Temperatures are $1.5 \mathrm{~K}, 3 \mathrm{~K}, 10 \mathrm{~K}, 20 \mathrm{~K}$, and $40 \mathrm{~K}$ from bottom to top. Graphs are offset by $100 \Omega$ for clarity. Upper inset: $1 / B$ position of the minima of the quantum oscillations. Filling factors $\nu$ correspond to the carrier density determined at high fields $\left(n_{s}\right.$ $=1.6 \times 10^{12} \mathrm{~cm}^{-2}$ ). A magnetic-field axis is included for guidance.

calculated numerically assuming a hard-wall potential. The brackets denote the average over phase space. Previously only chaotic trajectories were considered in the evaluation of the Kubo formula. ${ }^{9}$ Since the features we are interested in appear at relatively high magnetic-field values, most of the phase space is occupied by regular nonchaotic trajectories. Therefore we included both regular and chaotic trajectories in our calculations. By inverting the conductivity tensor we obtain the experimentally determined diagonal resistivity $\rho_{x x}$. The results of a simulation using the simple model with perfectly hard walls are shown in Fig. 3. The calculated traces are remarkably close to the experimental results: the additional structure between 1.5 and $3.5 \mathrm{~T}$ was nicely reproduced. The calculation shows a double peak structure (with maxima at $\sim 2.4$ and $\sim 3.3 \mathrm{~T}$ ), which is best seen in the experimental traces at temperatures above $10 \mathrm{~K}$ where the quantum oscillations are already strongly damped. We will address the double peak structure below.

To obtain information about the types of trajectories involved in the observed magnetoresistance anomalies we analyzed the Poincaré sections at different magnetic field values. This is done by injecting electrons at $x=0$ (between two antidots; see left-hand side of the lower part of Fig. 4) with velocity $v_{y}$ into the lattice and recording the $v_{y}$ and $y$ values of the injected electrons intersecting the line $x \bmod a=0$. To take into account possible soft-wall effects we assumed here an antidot potential of the form

$$
V(x, y)=V_{0}\left(\cos \frac{\pi x}{a} \cos \frac{\pi y}{a}\right)^{\beta}
$$




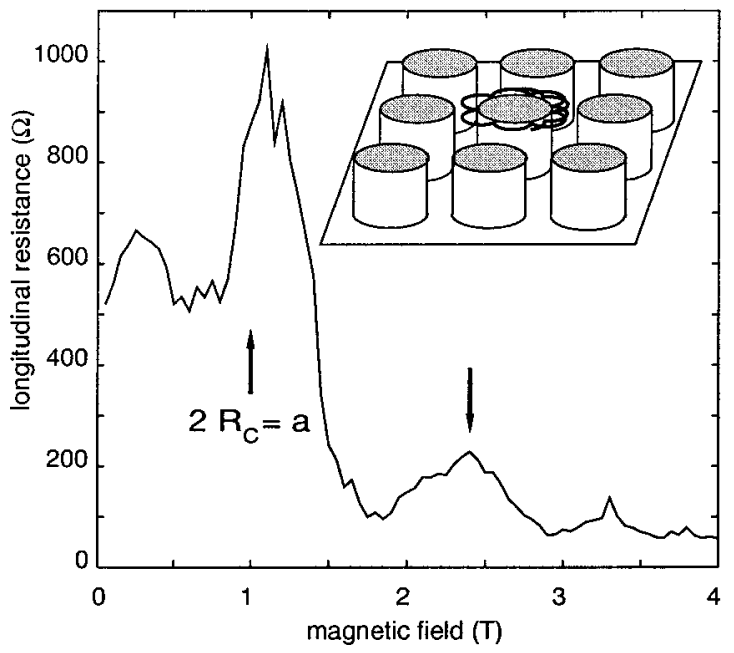

FIG. 3. Magnetoresistance traces obtained with a simple simulation based on the Kubo formula. The commensurability condition $2 R_{c}=a$ is shown for $n_{s}=1.3 \times 10^{12} \mathrm{~cm}^{-2}$ used in the calculation. Inset: A sketch of the hard-wall potential and a rosette-shaped orbit encircling an antidot. Its magnetic-field position is marked with an arrow in the large graph.

with $\beta=16$. The trajectories and velocities of the injected electrons were obtained by solving the classical equations of motion numerically at several magnetic fields (indicated with letters a-d in Figs. 1 and 4).

At about $1 \mathrm{~T}$ (letter a), the commensurability condition $2 R_{c}=a$ is satisfied, and the Poincare section exhibits a stable
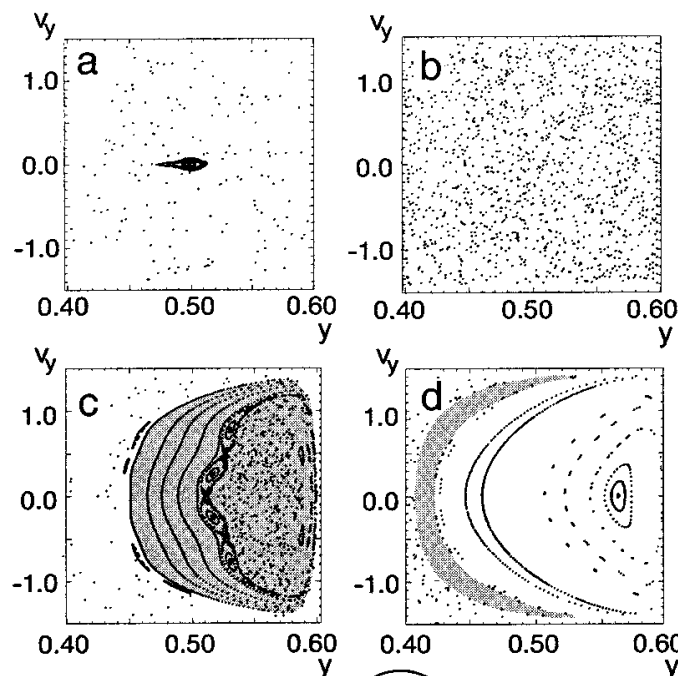

y
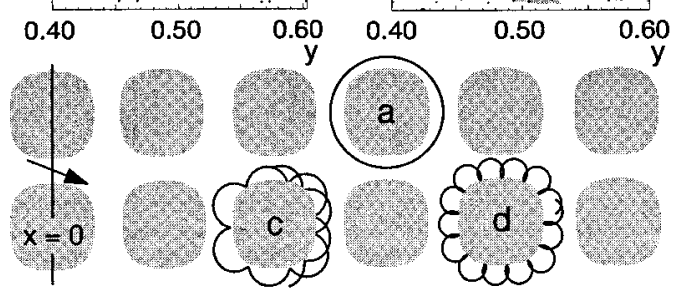

FIG. 4. Poincaré sections at $B$ fields labeled from a-d in Fig. 1. $y$ and $v_{y}$ are given in units of the lattice period $a$ and $\sqrt{2} v_{F}$, respectively. Trajectories shown below correspond to shaded areas in the Poincare sections. Note that for the highest magnetic field (d) the largest part of phase space is still occupied by stable orbits, only the orbits in the shaded region are unstable in an external electric field. Lower left corner: position of the Poincare plane. island [see Fig. 4(a)] belonging to cyclotron orbits around one antidot. This leads to the fundamental commensurability peak in $\rho_{x x}$. The minimum in the magnetoresistance at $1.9 \mathrm{~T}$ (letter $\mathrm{b}$ ) is due to the fact that the large antidots leave no room for stable trajectories. This minimum is not found in antidot lattices with smaller AD diameters. If the magnetic field gets higher, the Poincare section c shows that a large fraction of phase space is occupied by periodic and quasiperiodic trajectories. These are rosette-shaped orbits, which encircle one antidot and remain stationary even if an electric field is applied in the simulation, giving rise to a maximum in the magnetoresistance around $2.5 \mathrm{~T}$. The situation is quite similar to pinned orbits, which are responsible for the commensurability peak at $2 R_{c}=a$. A more detailed analysis of further phase space portraits between $2.5 \mathrm{~T}$ and $4 \mathrm{~T}$ suggests the following explanation for the double peak structure: Going to higher fields, stable islands of orbits that are reflected four times when surrounding one $\mathrm{AD}$ decrease in size, whereas the phase volume of orbits with eight reflections increases. This transition might be associated with the double peak structure seen both in experiment and calculation. The high-field behavior (letter $\mathrm{d}, B=4 \mathrm{~T}$ ) of the classical structure is governed by the formation of unstable rosette-shaped orbits. Instead of being composed more or less of semicircles, the small cyclotron diameter at high fields permits orbits which resemble circles revolving around one antidot. These orbits tend to drift perpendicular to an electric field, thus reducing $\rho_{x x}$, even though the stable orbits still occupy a large volume in phase space.

In antidot arrays, the underlying classical dynamics is also reflected in quantum oscillations superimposed upon the classical features. ${ }^{14}$ Here, we also find weak quantum oscillations imposed upon the classical double peak structure, which was ascribed to rosette-shaped orbits. These oscillations are suppressed at temperatures above a few Kelvin (see lower inset of Fig. 2), while the classical features survive up to temperatures of $40 \mathrm{~K}$ where they are blurred as the $\mathrm{GaSb}$ substrate becomes conducting. Compared to the $\mathrm{SdH}$ oscillations at high fields, the amplitude of the quantum oscillations is strongly supppressed.

While the periodicity of these oscillations is similar to the period of the $\mathrm{SdH}$ oscillations at higher magnetic field, there is a distinct transition between the high-field oscillations and the ones superimposed upon the classical maximum. This can be seen in the upper inset of Fig. 2 where the filling factor $\nu$ is plotted versus $1 / B$. At around $4 \mathrm{~T}\left(2 R_{c} \sim b\right)$ there is a pronounced kink in the otherwise linear slope which is observed at the transition between the classical peak regime and the quantum mechanical regime in which Shubnikov-de Haas oscillations dominate. We speculate that the supressed quantum oscillations stem from quantization of rosetteshaped orbits, since they dominate phase space in this intermediate magnetic field range. This has yet to be demonstrated by semiclassical calculations, which is beyond the scope of this work.

Once the magnetic field exceeds $3.5 \mathrm{~T}$, the amplitude of the $\mathrm{SdH}$ oscillations increases drastically. At magnetic fields below this threshold, the cyclotron diameter exceeds the width of the constriction between adjacent antidots. Therefore, electrons can easily backscatter from one edge of the sample to the other by hopping from $\mathrm{AD}$ to $\mathrm{AD}$. As soon as 
the cyclotron diameter becomes smaller, this backscattering process is no longer possible and the $\mathrm{SdH}$ oscillations become much more pronounced. The importance of edge channel transport in this field range was also shown by a fully quantum mechanical calculation. ${ }^{15}$

From the magnetic-field position of this point (marked by vertical lines in Fig. 2), we determine this critical cyclotron diameter to be $110 \mathrm{~nm}$. If we assume a very steep antidot potential, this leads to an antidot diameter of $270 \mathrm{~nm}$ that is slightly larger than the lithographic diameter of $250 \mathrm{~nm}$. Since on InAs surfaces the Fermi level is pinned in the conduction band, ${ }^{3}$ one would not expect a depleted region enlarging the antidots. However, the exact pinning position of the Fermi level depends on the detailed conditions of the etch-exposed surfaces and may well be different from the bulk Fermi level in the $2 \mathrm{DEG} .{ }^{16}$ Consequently, the enhance- ment of the antidot diameter can be due to a softer potential.

In summary, we fabricated and measured antidot arrays on InAs/GaSb with large AD diameters. We find a broad maximum in the magnetoresistance at intermediate magnetic fields characterized by $R_{c} \sim b$ as lower and $2 R_{c} \sim b$ as upper edge, which we show to be of classical origin. The trajectories responsible for this maximum are identified to be orbits involving multiple reflections on a single $\mathrm{AD}$. We also observe weak quantum oscillations on this maximum, which we speculate to originate from the same class of orbits. Finally, we estimate the effective diameter of the AD's and find that it is comparable with the lithographic diameter.

We would like to thank D. Heisenberg for helpful discussions. The authors acknowledge the financial support of the German Bundesministerium für Bildung und Forschung under Contract No. 01 BM 622/5.
*Electronic address: jonathan.eroms@ @hysik.uni-regensburg.de

${ }^{1}$ Mesoscopic Physics and Electronics, edited by T. Ando, Y. Arakawa, K. Furuya, S. Komiyama, and H. Nakashima (SpringerVerlag, Berlin, 1998).

${ }^{2}$ D. Weiss et al., Phys. Rev. Lett. 66, 2790 (1991).

${ }^{3}$ L. Ö. Olsson et al., Phys. Rev. Lett. 76, 3626 (1996).

${ }^{4}$ M. Altarelli, J. C. Maan, L. L. Chang, and L. Esaki, Phys. Rev. B 35, 9867 (1987).

${ }^{5}$ C. Nguyen, B. Brar, H. Kroemer, and J. H. English, Appl. Phys. Lett. 60, 1854 (1992).

${ }^{6}$ C. Nguyen, B. Brar, and H. Kroemer, J. Vac. Sci. Technol. B 11, 1706 (1993).

${ }^{7} \mathrm{H}_{3} \mathrm{PO}_{4}: \mathrm{H}_{2} \mathrm{O}_{2}: \mathrm{H}_{2} \mathrm{O}=1: 1: 12$.
${ }^{8}$ F.-C. Wang et al., Appl. Phys. Lett. 69, 1417 (1996).

${ }^{9}$ R. Fleischmann, T. Geisel, and R. Ketzmerick, Phys. Rev. Lett. 68, 1367 (1992).

${ }^{10}$ D. Többen et al., Phys. Rev. B 50, 8853 (1994).

${ }^{11}$ A. Lorke, S. Wimmer, B. Jager, and J. P. Kotthaus, Physica B 249-251, 312 (1998).

${ }^{12}$ C. Gould et al., Phys. Rev. B 51, 11213 (1995).

${ }^{13}$ R. Kubo, J. Phys. Soc. Jpn. 12, 570 (1957).

${ }^{14}$ D. Weiss et al., Phys. Rev. Lett. 70, 4118 (1993).

${ }^{15}$ I. V. Zozoulenko, F. A. Maaø, and E. H. Hauge, Phys. Rev. B 53, 7975 (1996).

${ }^{16}$ B. J. van Wees et al., Phys. Rev. B 51, 7973 (1995). 